\title{
URAT1 gene polymorphisms influence uricosuric action of losartan in hypertensive patients with hyperuricemia
}

\begin{abstract}
Aims: To explore the effect of urate transporter 1 (URAT1) polymorphisms on the hypertensive patients with hyperuricemia and the uricosuric action of losartan therapy among hypertensive patients with hyperuricemia. Methods: 101 hypertensive patients with hyperuricemia were detected the genotypes of URAT1 rs1529909 and rs3825016 and undergo a 2-weeks following losartan treatment. Before and after treatment, serum uric acid (SUA) and other clinical data were compared between different genotypes of URAT1 patients. Results: The frequency of rs3825016 (C/T) CT genotype was significant higher in the hypertensive patients with hyperuricemia than that in the healthy controls ( 32.7 vs $18.8 \% ; p=0.02$ ). After lorsatan treatment, the patients with the rs3825016 (C/T) or rs1529909 (T/C) mutant genotypes had lower decreased value (DV) of SUA compared with the patients who are wild-type of the variant $(p=0.001$ and $p<0.001$, respectively). Combined the two variants together, the DV of SUA in two variants both wild-type patients higher than that in the two variants mutant patients $(p<0.0001)$. Conclusion: These results suggest that URAT1 rs3825016 and rs1529909 polymorphisms influence the uricosuric action of losartan.
\end{abstract}

Original submitted 20 August 2014; Revision submitted 15 April 2015

Keywords: Iorsatan $\bullet$ polymorphism $\bullet$ serum uric acid $\bullet$ URAT1 $\bullet$ uricosuric action

Previous clinical reports indicate losar$\tan$ as an antihypertension drug has a significant uricosuric action in hypertensive patients [1-5]. Owing to the specific benefit on urate metabolism, losartan has been known to ameliorate diuretic-induced hyperuricemia $[6,7]$. The mechanism of its special effect has been reported to result from the influence of mRNA level of urate transporter 1(URAT1) gene to inhibit the urate/anion transport in brush-border cells of the renal proximal tubules [8-12].

The urate/anion transporter appears to be essential for urate reabsorption because only urate-reabsorbing species possess exchangers with affinity for urate [13]. Up to now, several urate/anion transporters such as breast cancer resistance protein (BCRP/ABCG2), glucose transporter family 2, member 9 (SLC2A9), urate transporter 1 (URAT1:
SLC22A12) have been identified in the human kidney [6,9,14], however, only URAT1 was found to be the target of losartan to reduce the serum uric acid [1,15]. URAT1 localized to the apical side of the proximal tubule in the human kidney has been identified in 2002 [9]. Functional studies using a Xenopus oocytes expression system revealed that the properties of urate transport by URAT1 are comparable to those in human renal brush border membrane vesicles and that URAT1 is a high-affinity urate/anion exchanger $(\mathrm{Km}: 371 \mathrm{mmol} / \mathrm{l})[9,13]$. In vivo, some findings suggested that losartan inhibited URATI and thereby reduced serum uric acid in patients with hypertension $[15,16]$.

Surprisingly, the uricosuric action of losartan is different in patients and the mechanism of its individual uricosuric action is still not clear. Few studies inves-
Hong Sun $\neq 1,2$, Qiang $\mathrm{Qu}^{\ddagger 1,13}$, Jian $\mathrm{Qu}^{\ddagger 1}$, , Xiao-Ya Lou', Yan Peng', Ying Zeng' \& Guo Wang ${ }^{*, 1}$ 'Department of Clinical Pharmacology, Institute of Clinical Pharmacology, Hunan Key Laboratory of Pharmacogenetics, Central South University Xiangya School of Medicine, Changsha 410078, PR China ${ }^{2}$ Department of Pharmacy, Provincial Clinical College of Fujian Medical University, Fujian Provincial Hospital, Fuzhou 350001, PR China

${ }^{3}$ Department of Pharmacy, Xiangya Hospital, Central South University,

Changsha 410008, PR China

*Author for correspondence:

Tel.: +8673184805380

Fax: +8673182354476 wangguo32@126.com

${ }^{\ddagger}$ Authors contributed equally
Future
Medicine 
tigate whether the variants of URAT1 influence the uricosuric action of losartan. In view of the fact that the URAT1 plays an important role in the uricosuric action of losartan, we aimed to assess not only the association between URAT1 rs1529909 (T/C)/ rs3825016 (C/T) polymorphisms and serum uric acid but also the impact of these variants on the uricosuric efficacy of losartan in hypertensive patients with hyperuricemia.

\section{Patients \& methods}

\section{Subjects}

A total of 101 hypertensive patients with hyperuricemia (mean age: $64 \pm 15$ years, 53 female patients and 48 male patients) and 117 healthy controls (mean age: $64.6 \pm 12.9$ years, 57 female subjects and 60 male subjects) were recruited and grouped by the genotypes of URAT1 rs3825016 (C/T) and rs1529909 (T/C) polymorphisms from Xiangya Hospital. Healthy controls were those people who have no hypertension and hyperuricemia, as well other illnesses. Patients who had a systolic blood pressure (SBP) $>140 \mathrm{~mm} \mathrm{Hg}$ and/or a diastolic blood pressure (DBP) $>90 \mathrm{~mm} \mathrm{Hg}$ were diagnosed as hypertensive [15]. Hyperuricemia was defined as patients having a serum uric acid level more than $7 \mathrm{mg} / \mathrm{dl}(416 \mathrm{umol} / \mathrm{l})$ in men or $6 \mathrm{mg} / \mathrm{dl}$ (357 umol/l) in woman [17]. Patients with any of the following were excluded from the study: history of gout or renal lithiasis and other metabolic disease and complications; drugs or alcohol abuse and use other drugs influencing the serum uric acid levels or urine volume; pregnancy or lactation. All subjects were evaluated with respect to their medical history and underwent a physical examination and routine clinical laboratory tests. Written informed consent was obtained from each subject before the start of this study.

A total of 101 hypertensive patients with hyperuricemia with various URAT1 rs3825016 (C/T) and rs1529909 (T/C) genotypes were selected to take a daily dosage of $100 \mathrm{mg}$ losartan orally for two consecutive weeks. The study was performed according to the principles of the Declaration of Helsinki (revision of Edinburgh 2000) and a clinical study admission (the registration number: ChiCTR-RCH-12002706) was approved by Chinese Clinical Trail Register. The study protocol was approved by the Ethics Committee of Xiangya School of Medicine, Central South University, China.

\section{Clinical laboratory tests}

Height, body weight, BMI and blood pressure were measured. Venous blood samples were obtained to detect serum biochemical parameters including blood urea nitrogen (BUN), serum creatinine (Cr), serum uric acid (SUA), electrolytes, total choles- terol (CHOL), high-density lipoprotein cholesterol (HDL-c), low-density lipoprotein cholesterol (LDL-c) and triglyceride (TG).

\section{Genotyping procedures for URAT1 rs3825016 (C/T) \& rs1529909 (T/C) polymorphisms}

Genomic DNA was isolated from peripheral blood leukocytes using phenol-chloroform followed by ethanol precipitation and then stored at $4^{\circ} \mathrm{C}$ until use. The primer pairs used in the amplification of the URAT1 rs 3825016 $(\mathrm{C} / \mathrm{T})$ locus were sense primer: 5'-CCTCACGCGGCCTCAGGGCCCAGTT-3', antisense primer: 5'-GGGTCCCTCCCAGGACTGGACCTTT-3'. The primer pairs used for rs1529909 (T/C) were sense primer: 5'-ATGGCTGAAAGGCAAGACC-3', antisense primer: 5'- ACAGCATCCCAACCACAATC-3'. The PCR amplifications were carried out in a total volume of $25 \mu \mathrm{l}$ containing $2.5 \mu \mathrm{l} 10 \times$ PCR buffer (with $\mathrm{MgCl}_{2}$ ) (Takara, Dalian, China), $0.2 \mathrm{mmol} / \mathrm{l}$ of each dNTP (Takara), $0.4 \mathrm{mmol} / \mathrm{l}$ of each primer, $200 \mathrm{ng}$ of genomic DNA as a template, and $1 \mathrm{U}$ Taq polymerase (Takara). The PCR conditions were as follows: predenaturing at $94^{\circ} \mathrm{C}$ for $5 \mathrm{~min}$, followed by 30 cycles of denaturing at $94^{\circ} \mathrm{C}$ for $30 \mathrm{~s}$, annealing for $30 \mathrm{~s}$ and extending at $72^{\circ} \mathrm{C}$ for $30 \mathrm{~s}$, and finally extending at $72^{\circ} \mathrm{C}$ for $8 \mathrm{~min}$. The annealing temperatures for the two polymorphisms were $71.3^{\circ} \mathrm{C}$ and $54^{\circ} \mathrm{C}$, respectively. The PCR products for URAT1 rs3825016 (C/T) were analyzed by direct sequencing and the PCR products for analysis of rs1529909 (T/C) polymorphism was digested by VAN91 I (Takara) and then be sequenced to confirm the genotype. The restriction enzyme digests for URAT1 rs1529909 (T/C) polymorphisms were separated on $3 \%$ agarose gels and visualized by ethidium bromide staining.

\section{Statistical analysis}

All statistical analyses were carried out using SPSS software (version 11.0 for Windows; SPSS, Chicago, IL, USA). Hardy-Weinberg equilibrium was assessed using the $\chi^{2}$ test in patients sample and healthy controls. Comparisons of baseline characteristics in hypertensive patients with hyperuricemia and healthy controls were carried out using the paired Student's t-test or Mann-Whitney U-test. Comparisons of the frequencies of genotypes and alleles were analyzed using the $\chi^{2}$ test. Baseline characteristics among genotypes were compared using one-way analysis of variance or Mann-Whitney U-test. To compare the effects of losartan on the metabolic parameters of subjects with the various genotypes, independent-samples t-tests and nonparametric Mann-Whitney U-test were used. Data are presented as mean \pm SD unless indicated otherwise. $\mathrm{p}<0.05$ was considered statistically significant. 


\section{Results}

Clinical characteristics of subjects

The URAT1 polymorphism genotypes were determined in 101 hypertensive patients with hyperuricemia and in 117 healthy controls. The clinical characteristics of the subjects are summarized in Table 1. There were significant differences between the hypertensive patients with hyperuricemia and the healthy controls in BMI $(p=0.001)$, serum uric acid $(p<0.001)$, creatinine $(\mathrm{Cr})(\mathrm{p}<0.001)$, blood urea nitrogen $(\mathrm{BUN})$ $(p<0.001)$, plasma concentration of high-density lipoprotein cholesterol (HDL-c) $(\mathrm{p}=0.008)$, systolic blood pressure (SBP) $(\mathrm{p}<0.001)$, diastolic blood pressure $(\mathrm{DBP})(\mathrm{p}<0.001)$ and low-density lipoprotein cholesterol (LDL-c) $(\mathrm{p}=0.014)$ levels. No significant differences between the two groups were seen with gender distribution, age, blood urea nitrogen/creatinine (BUN/Cr) and total cholesterol (CHOL).

\section{Genotype analysis \& allelic frequencies}

The allelic frequencies of the URAT1 rs3825016 $(\mathrm{C} / \mathrm{T})$ and $\mathrm{rs} 1529909$ (T/C) in the hypertensive patients with hyperuricemia and in the healthy controls are shown in Table 2. The $\mathrm{C}$ allelic frequencies of the URAT1 rs1529909 (T/C) were $33.7 \%$ in the hypertensive patients with hyperuricemia and $32.1 \%$ in the healthy controls, and the $\mathrm{T}$ allelic frequencies of the URAT1 rs3825016 (C/T) were 20.3 and $14.5 \%$ in the hypertensive patients with hyperuricemia and the healthy controls, respectively. In this study, we found the frequency of rs3825016 (C/T) CT genotype was significant higher in hypertensive patients with hyperuricemia than that in healthy controls (32.7 vs $18.8 \%$, $\mathrm{p}=0.02)$.

\section{Comparison of baseline parameters of the} URAT1 rs3825016 (C/T) \& rs1529909 (T/C) genotypes among the hypertensive patients with hyperuricemia

The baseline clinical characteristics of 101 hypertensive patients with hyperuricemia were measured before losratan therapy. There were no differences among the patients who had various genotypes of URAT1 in all baseline parameters (Table 3).

\section{Influence of URAT1 rs3825016 \& rs1529909}

(T/C) polymorphisms on the uricosuric action of losartan in hypertensive patients with hyperuricemia

Losartan significantly decreased the values of SUA $(p<0.001)$, Cr $(p<0.001)$, BUN $(p<0.001)$, SBP $(\mathrm{p}<0.001)$ and DBP $(\mathrm{p}<0.001)$ in hypertensive patients with hyperuricemia after 2 weeks of losartan treatment (Table 4). Moreover, after losartan treatment, the decreased value and percentage of SUA in patients with the URAT1 rs3825016 (C/T) polymorphism CT+TT genotypes $(\mathrm{n}=37)$ were significantly lower than those in patients with CC genotype $(n=64$; all $p<0.01)$.

Table 1. Clinical characteristics of hypertensive patients with hyperuricemia and healthy controls.

\begin{tabular}{|c|c|c|c|}
\hline Parameter & $\begin{array}{l}\text { Healthy controls } \\
(n=117)\end{array}$ & $\begin{array}{l}\text { Hypertensive patients with } \\
\text { hyperuricemia }(n=101)\end{array}$ & p-value \\
\hline Male/female & $60 / 57$ & $48 / 53$ & 0.580 \\
\hline Age $\left(\right.$ years) ${ }^{\dagger}$ & $64.6 \pm 12.9$ & $64.0 \pm 15.0$ & 0.289 \\
\hline BMI $\left(\mathrm{kg} / \mathrm{m}^{2}\right)$ & $23.5 \pm 2.3$ & $22.9 \pm 2.4$ & 0.001 \\
\hline SUA (umol/l) & $291.9 \pm 58.9$ & $503.04 \pm 81.27$ & $<0.0001$ \\
\hline $\mathrm{Cr}(\text { umol/l })^{\dagger}$ & $46 \pm 20.9$ & $94.32 \pm 18.65$ & $<0.0001$ \\
\hline BUN (mmol/l) & $5.1 \pm 1.4$ & $6.73 \pm 2.68$ & $<0.0001$ \\
\hline $\mathrm{BUN} / \mathrm{Cr}$ & $70.67 \pm 21.29$ & $72.36 \pm 26.88$ & 0.792 \\
\hline LDL-c (mmol/l) & $2.7 \pm 0.99$ & $3.08 \pm 1.11$ & 0.014 \\
\hline HDL-c (mmol/l) & $1.3 \pm 0.44$ & $1.15 \pm 0.35$ & 0.008 \\
\hline $\mathrm{CHOL}(\mathrm{mmol} / \mathrm{l})$ & $4.8 \pm 0.97$ & $4.71 \pm 1.37$ & 0.222 \\
\hline TG (mmol/l) & $1.6 \pm 1.09$ & $1.73 \pm 0.83$ & 0.067 \\
\hline $\mathrm{SBP}(\mathrm{mmHg})$ & $112 \pm 13.9$ & $138.60 \pm 25.3$ & $<0.0001$ \\
\hline $\mathrm{DBP}(\mathrm{mmHg})$ & $74 \pm 12.7$ & $80.60 \pm 14.5$ & $<0.0001$ \\
\hline \multicolumn{4}{|c|}{$\begin{array}{l}\text { Values in bold }=p \text {-values }<0.05 \text { and significant difference. } \\
\text { Data are shown as mean } \pm \text { standard deviation. } \\
\text { +Compared with independent-samples t-test, others with Mann-Whitney U-test. } \\
\text { BUN: Blood urea nitrogen; BUN/Cr: Blood urea nitrogen/creatinine; CHOL: Cholesterol; Cr: Creatinine; DBP: Diastolic blood pressure; } \\
\text { HDL-c: High-density lipoprotein cholesterol; LDL-C: Low-density lipoprotein cholesterol; SBP: Systolic blood pressure; SUA: Serum uric acid; } \\
\text { TG: Triglyceride. }\end{array}$} \\
\hline
\end{tabular}




\begin{tabular}{|c|c|c|c|}
\hline Genotype & $\begin{array}{l}\text { Healthy controls } \\
(n=117)\end{array}$ & $\begin{array}{l}\text { Hypertensive patients } \\
\text { with hyperuricemia } \\
(n=101)\end{array}$ & $p$-value \\
\hline \multirow[t]{5}{*}{ URAT1 rs1529909 (T/C) } & T $159(67.9 \%)$ & T $134(66.3 \%)$ & Reference \\
\hline & C $75(32.1 \%)$ & C $68(33.7 \%)$ & 0.72 \\
\hline & TT 52 (44.5\%) & TT $46(45.5 \%)$ & Reference \\
\hline & СТ 55 (47\%) & CT $42(41.6 \%)$ & 0.61 \\
\hline & CC $10(8.5 \%)$ & CC $13(12.9 \%)$ & 0.51 \\
\hline \multirow[t]{5}{*}{ URAT1 rs3825016 (C/T) } & C $200(85.5 \%)$ & C $161(79.7 \%)$ & Reference \\
\hline & T $34(14.5 \%)$ & T $41(20.3 \%)$ & 0.11 \\
\hline & CC $89(76.1 \%)$ & CC $64(63.4 \%)$ & Reference \\
\hline & CT $22(18.8 \%)$ & CT $33(32.7 \%)$ & 0.02 \\
\hline & TT $6(5.1 \%)$ & TT $4(3.9 \%)$ & 0.91 \\
\hline
\end{tabular}

The URAT1 rs1529909 (T/C) polymorphism was also associated with a decrease of losartan efficacy. Patients with rs1529909 (T/C) CC+CT genotypes $(\mathrm{n}=55)$ had a significant lower the decreased value and decreased percentage of SUA, Cr and BUN/Cr as compared with patients with the TT genotype $(\mathrm{n}=46)($ all $\mathrm{p}<0.05)$ (Table 5).

URAT1 rs3825016 (C/T) \& rs1529909 (T/C) joint influence on uricosuric action of losartan in hypertensive patients with hyperuricemia We further assessed the joint influence of the URAT1 rs3825016 (C/T) and rs1529909 (T/C) variants on uricosuric action of losartan in hypertensive patients with hyperuricemia. We investigated the differences of parameters between the rs3825016 (C/T)-rs1529909 (T/C) CC-TT genotype group $(\mathrm{n}=37)$ and $\mathrm{rs} 3825016$ (C/T)-rs1529909 (T/C) mutation group $(\mathrm{n}=64)$. Combined the two variants together, the decreased value (DV) and decreased percentage (DP) of SUA in two variants both wild-type patients was higher than that in the two variants mutant patients $(\mathrm{p}<0.0001)$ (Figures 1 \& 2).

\section{Discussion}

In this study, we found that URAT1 rs3825016 (C/T) and rs1529909 (T/C) polymorphisms influenced the uricosuric action of losartan in hypertensive patients with hyperuricemia. The patients who carried URAT1 rs3825016 (C/T) and rs1529909 (T/C) wild-type alleles showed a more prominent decrease of serum uric acid level than the decrease in the patients who carried URAT1 rs3825016 or rs1529909 mutant alleles.

As the angiotensin II receptor blocker, lorsatan can decreased the blood press. Moreover, it can decrease serum uric acid level in a dose-dependent manner with single doses ranging from $25-200 \mathrm{mg}$ [2]. Sweet et al. demonstrated that the uricosuric effect of losartan was due to the parent compound and not to the metabolite EXP-3174 [18]. Most previous studies focused on reducing blood pressure of lorsatan, but few studies investigated its uricosuric action. URAT1 was involved in the metabolism of serum uric acid and losartan could decrease SUA via inhibiting URAT1 transporter and decreasing the level of its mRNA $[12,15,19,20]$. The uricosuric efficacy of losartan presents individual differences in patients. Hence, URAT1 is probably involved in effecting losartan uricosuric action.

Previous research found that URAT1 variants influence the renal uric acid excretion [21]. The genotype frequencies in controls and in German Caucasian hyperuricemia patients with reduced uric acid excretion were significantly different and the $258 \mathrm{~T}$ allele was associated with a reduction of the risk for reduced the fractional excretion of uric acid (FEUA) [21]. However, Iwai et al. investigated the effects of some polymorphisms of the URAT1, including rs3825016 (C/T), on serum uric acid levels in a Japanese population and failed to observe any effect on serum uric acid level [22]. Genotype frequencies were completely different between the two populations. The prevalence of the CC, CT and TT polymorphisms in the Japanese patients was $72.5,27.5$ and $0.0 \%$, respectively, compared with $14.9,41.9$ and $43.2 \%$ in the German cohort. In our study, the frequencies of three genotypes were 63.4, 32.7 and $3.9 \%$ in patients, respectively. We found the frequency of rs3825016 (C/T) CT genotype was significant higher in hypertensive patients with hyperuricemia than in healthy controls (32.7 vs $18.8 \%, \mathrm{p}=0.02$ ). Our results were consistent with the German cohort results but not consistent with the Japa- 


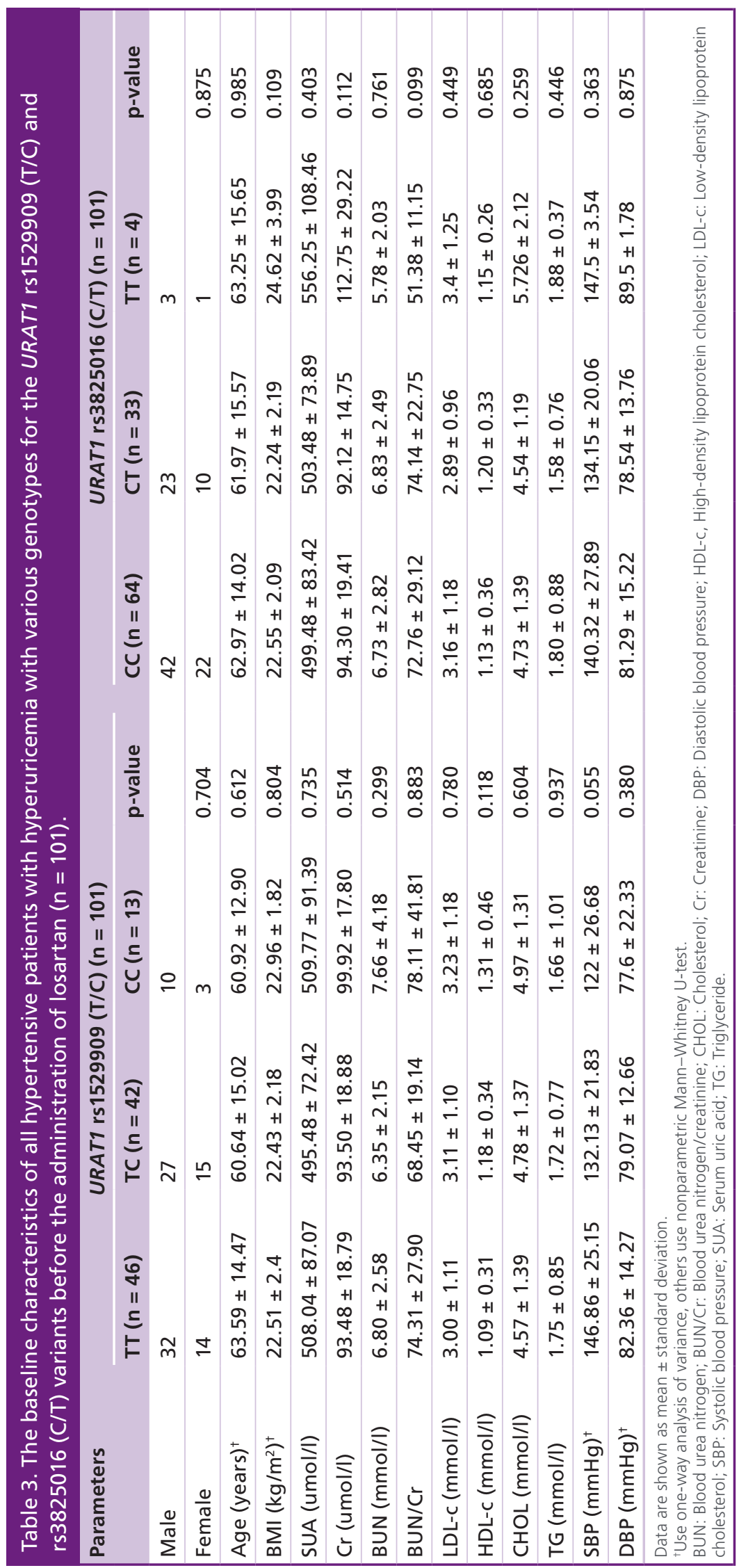




\begin{tabular}{|c|c|c|c|}
\hline Parameters & Before & After & $p$-value \\
\hline SUA (umol/l) & $503.04 \pm 81.27$ & $449.96 \pm 107.6$ & $<0.0001$ \\
\hline $\mathrm{Cr}$ (umol/l) & $94.32 \pm 18.65$ & $87.60 \pm 20.37$ & $<0.0001$ \\
\hline BUN (mmol/l) & $6.73 \pm 2.68$ & $5.80 \pm 2.50$ & $<0.0001$ \\
\hline $\mathrm{BUN} / \mathrm{Cr}$ & $72.36 \pm 26.88$ & $66.84 \pm 24.82$ & 0.068 \\
\hline LDL (mmol/l) & $3.08 \pm 1.11$ & $3.09 \pm 1.34$ & 0.040 \\
\hline HDL (mmol/l) & $1.15 \pm 0.35$ & $1.05 \pm 0.18$ & 0.061 \\
\hline CHOL (mmol/l) & $4.71 \pm 1.37$ & $4.82 \pm 1.59$ & 0.943 \\
\hline TG $(\mathrm{mmol} / \mathrm{l})$ & $1.73 \pm 0.83$ & $1.93 \pm 1.32$ & 0.631 \\
\hline $\mathrm{SBP}(\mathrm{mmHg})^{\dagger}$ & $138.6 \pm 25.3$ & $118.0 \pm 10.5$ & $<0.0001$ \\
\hline DBP $(\mathrm{mmHg})^{\dagger}$ & $80.6 \pm 14.5$ & $70.1 \pm 7.7$ & $<0.0001$ \\
\hline \multicolumn{4}{|c|}{$\begin{array}{l}\text { Values in bold }=p \text {-values }<0.05 \text { and significant difference. } \\
\text { Data are shown as mean } \pm \text { standard deviation. } \\
\text { tUse paired Student's t-test, others use nonparametric Mann-Whitney U-test. } \\
\text { BUN: Blood urea nitrogen; BUN/Cr: Blood urea nitrogen/creatinine; CHOL: Cholesterol; Cr: Creatinine; DBP: Diastolic blood pressure; } \\
\text { HDL-C: High-density lipoprotein cholesterol; LDL-c: Low-density lipoprotein cholesterol; SBP: Systolic blood pressure; SUA: Serum uric acid; } \\
\text { TG: Triglyceride. }\end{array}$} \\
\hline
\end{tabular}

nese research. Jang et al. reported the rs1529909 (T/C) polymorphism may be involved in renal urate handling and the concentration of serum uric acid in a study with 196 healthy male Korean subjects [23]. However, in our study we found no difference of rs1529909 (T/C) polymorphism between healthy control and hypertensive patients with hyperuricemia and no difference on serum uric acid among rs1529909 (T/C) variant in hypertensive patients with hyperuricemia. The cause leading to different results is probably a difference in ethnicity and also a too small sample number Larger samples in different ethnicities will be needed to confirm the results.
It has been shown that losartan, not other ARBs, has a beneficial effect to reduce serum uric acid levels in several studies [24-26]. It seemed obvious that losartan was effective to reduce serum uric acid levels in the patients with higher serum uric acid levels. This effect was also confirmed in the present study. The data from our study showed that losartan could significantly decrease the levels of SUA, Cr, BUN, SBP and DBP in hypertensive patients with hyperuricemia after 2 weeks' treatment (Table 4). Firstly we compared the baseline parameters of URAT1 rs3825016 (C/T) and rs1529909 (T/C) genotypes among the hyperten-

\section{Table 5. Comparisons of decreased values and decreased percentages in hypertensive patients with hyperuricemia with different URAT1 rs1529909 (T/C) and rs3825016 (C/T) genotypes before and after losartan treatment.}

\begin{tabular}{|c|c|c|c|c|c|c|}
\hline \multirow[t]{2}{*}{ Parameter } & \multicolumn{3}{|c|}{ URAT1 rs1529909 (T/C) $(n=101)$} & \multicolumn{3}{|c|}{ URAT1 rs3825016 $(\mathrm{C} / \mathrm{T})(\mathrm{n}=101)$} \\
\hline & $\operatorname{TT}(n=46)$ & $T C+C C(n=55)$ & p-value & $\mathrm{CC}(n=64)$ & $C T+T T(n=37)$ & $p$-value \\
\hline SUA (umol/I) ${ }^{\dagger}$ & $110.57 \pm 69.25$ & $5.00 \pm 12.91$ & $<0.0001$ & $69.27 \pm 74.36$ & $25.08 \pm 55.23$ & 0.001 \\
\hline $\mathrm{BUN}^{+}$ & $0.82 \pm 1.58$ & $1.02 \pm 1.01$ & 0.664 & $0.84 \pm 1.41$ & $1.41 \pm 1.09$ & 0.631 \\
\hline $\mathrm{BUN} / \mathrm{Cr}^{\dagger}$ & $0.003 \pm 0.014$ & $0.007 \pm 0.012$ & 0.008 & $0.004 \pm 0.011$ & $0.007 \pm 0.013$ & 0.125 \\
\hline $\mathrm{Cr}(\mathrm{mmol} / \mathrm{l})^{\ddagger}$ & $8.67 \% \pm 14.83 \%$ & $5.75 \% \pm 7.44 \%$ & 0.004 & $8.07 \% \pm 11.95 \%$ & $5.35 \% \pm 10.44 \%$ & 0.084 \\
\hline $\mathrm{BUN}^{\ddagger}$ & $11.88 \% \pm 19.17 \%$ & $14.51 \% \pm 18.03 \%$ & 0.470 & $11.71 \% \pm 21.37 \%$ & $16.08 \% \pm 11.84 \%$ & 0.410 \\
\hline $\mathrm{BUN} / \mathrm{Cr}^{\ddagger}$ & $2.69 \% \pm 15.37 \%$ & $6.83 \% \pm 22.15 \%$ & 0.016 & $3.6 \% \pm 20.46 \%$ & $7.73 \% \pm 18.09 \%$ & 0.079 \\
\hline \multicolumn{7}{|c|}{$\begin{array}{l}\text { Values in bold }=p \text {-values }<0.05 \text { and significant difference. } \\
\text { p-values are determined by nonparametric Mann-Whitney U-test. } \\
\text { Data are shown as mean } \pm \text { standard deviation. } \\
\text { †Postadministration minus preadministration; decreased percentages. } \\
\text { †(Postadministration minus preadministration)/postadministration. } \\
\text { BUN: Blood urea nitrogen; BUN/Cr: Blood urea nitrogen/creatinine; CHOL: Cholesterol; Cr: Creatinine; DBP: Diastolic blood pressure; HDL-C: High-density lipoprotein } \\
\text { cholesterol; LDL-C: Low-density lipoprotein cholesterol; SBP: Systolic blood pressure; SUA: Serum uric acid; TG: Triglyceride. }\end{array}$} \\
\hline
\end{tabular}


(A)

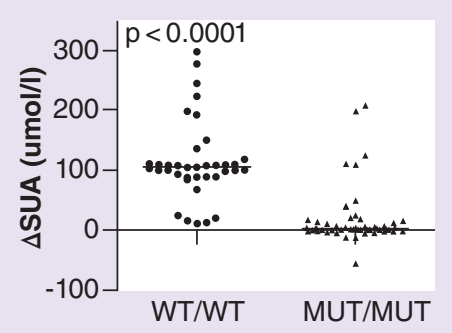

(B)

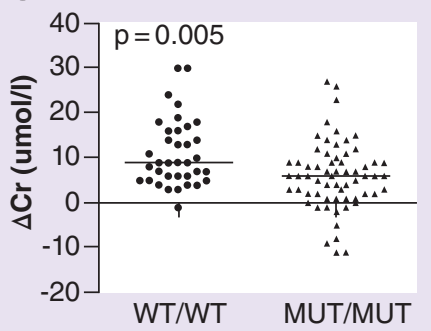

(C)

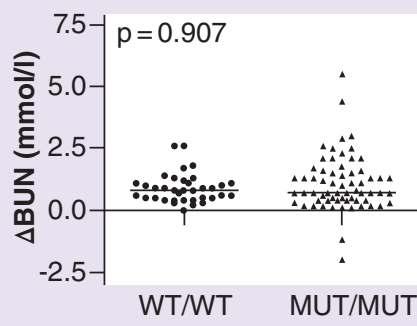

(D)

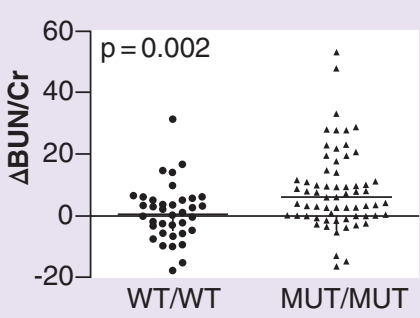

Figure 1. Comparisons of decreased values ( $\Delta=$ postadministration minus preadministration). (A) $\Delta S U A$, (B) $\Delta C r,(C) \Delta B U N$, (D) $\triangle B U N / C r$ between the WT and MUT of the URAT1 rs1529909 (T/C) and rs3825016 (C/T) patients after treatment with losartan. WT, URAT1 rs1529909 (T/C) and rs3825016 (C/T) TT-CC genotype patients; MUT, at least one mutant allelic patients.

$* \mathrm{p}<0.05$.

BUN: Blood urea nitrogen; BUN/Cr: Blood urea nitrogen/creatinine; CR: Creatinine; MUT: Mutant; SUA: Serum uric acid; WT: Wild-type.

sive patients with hyperuricemia to exclude the influence of URAT1 variants on baseline parameters and there were no difference among different genotypic patients on all parameters. After 2 weeks' losartan treatment, we observed that the patients with at least one $\mathrm{T}$ allele of the rs3825016 (C/T) polymorphism showed a more prominent decrease of serum uric acid level than the patients with the CC genotype; the patients with at least one C allele of the rs1529909 (T/C) polymorphism showed a more prominent decrease of Ur, Cr, BUN/Cr levels than the patients with the TT genotype. Moreover, when combined the two polymorphisms together, patients with mutant alleles have less uricosuric action of losartan than the patients who are wild-type of the two polymorphisms (Figures 1 \& 2).

URAT1 rs3825016 (C/T) polymorphism is located on exon 1 and although it is silent, we presumed this polymorphism is near the promoter and may have some promoter function and the variant possibly changes protein conformation and stability. rs1529909 (T/C) polymorphism is located on intron 4. Previous studies identified that URAT1 rs893006 polymorphism in the intron region that was heralded as a potent candidate gene of renal uric acid modulation and proposed the possibility of linkage disequilibrium for functional SNPs that affect the URAT1 molecule in the proximal tubule [23,27]. The SNP rs893006 in intron 4 is closely located to the SNP rs1529909 (T/C), therefore intron 4 of the URAT1 may contribute to the URAT1 molecule. In our study we indeed observed these two SNPs could significantly influence the uricosuric action of losartan and the reason could be their effect on the function of URAT1 inducing the capability of drug binding.

In conclusion, this study demonstrates that the rs3825016 (C/T) and the rs1529909 (T/C) variants of URAT1 influence the uricosuric action of losartan in hypertensive patients with hyperuricemia. They are potential genetic markers for the optimization of losartan's uricosuric action. Further studies are necessary to confirm our results in replicated larger populations.

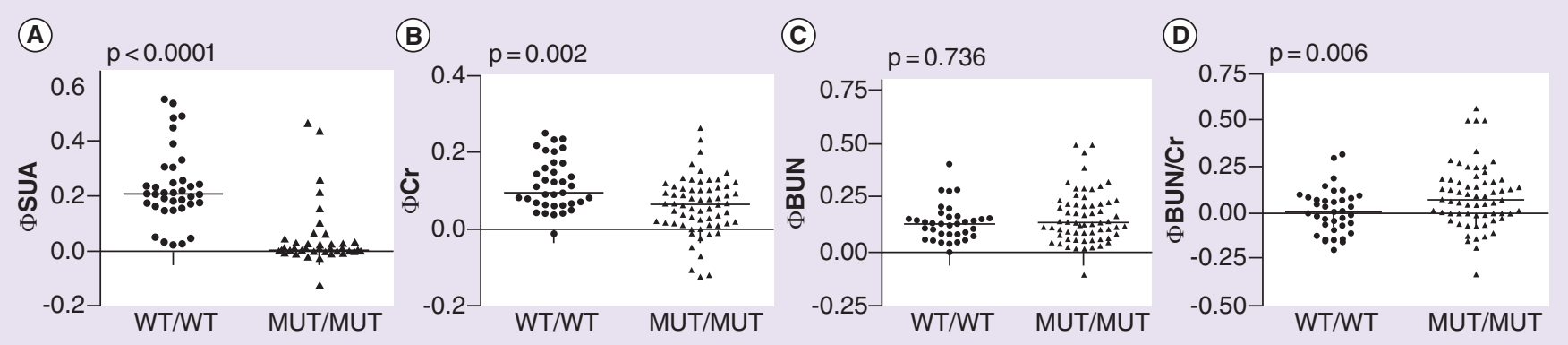

Figure 2. Comparisons of decreased percentage ( $\Phi=$ [postadministration minus preadministration]/postadministration).

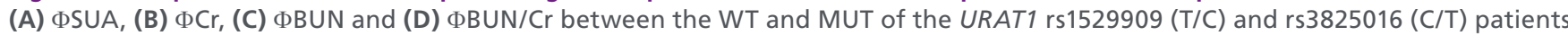
after treatment with losartan. WT, URAT1 rs1529909 (T/C) and rs3825016 (C/T) TT-CC genotype patients; MUT, at least one mutant allelic patients.

${ }^{*} p<0.05$.

BUN: Blood urea nitrogen; BUN/Cr: Blood urea nitrogen/creatinine; CR: Creatinine; MUT: Mutant; SUA: Serum uric acid; WT: Wild-type. 


\section{Future perspective}

Losartan as the angiotensin II receptor blocker has a special uricosuric action to reduce serum uric acid in hypertensive patients. We found that some urate transporter 1 gene polymorphisms influence the uricosuric action of losartan. Future work in this area should focus on whether there were more genes or more polymorphisms involved in the uricosuric action of losartan. The potential mechanism of these polymorphisms influence the uricosuric action of losartan could take advantage to individualized treatment for the hypertensive patients with hyperuricemia.

\section{Acknowledgements}

The authors thank all the funds that supported the study, and all patients who participated in the study.

Financial \& competing interests disclosure

The research project was supported by the Nature Science Foundation of Fujian Province (no. 2013J01364), the National Natural Science Foundation of China (no. 81072706), the Science and Technology Project of Hunan Province, China Under Grant (no. 2013FJ30), Research Innovation Foundation of Graduate Student in Hunan province, PRC (CX2011B056) and Hunan Provincial Innovation Foundation For Postgraduate (CX2013B102). The authors have no other relevant affiliations or financial involvement with any organization or entity with a financial interest in or financial conflict with the subject matter or materials discussed in the manuscript apart from those disclosed.

No writing assistance was utilized in the production of this manuscript.

Ethical conduct of research

The authors state that they have obtained appropriate institutional review board approval or have followed the principles outlined in the Declaration of Helsinki for all human or animal experimental investigations. In addition, for investigations involving human subjects, informed consent has been obtained from the participants involved.

\section{Open access}

This work is licensed under the Creative Commons Attribution-NonCommercial 3.0 Unported License. To view a copy of this license, visit http://creativecommons.org/licenses/bync-nd/3.0/

Executive summary

\section{Background}

- Losartan as the angiotensin II receptor blocker has a special uricosuric action to reduce serum uric acid in hypertensive patients. Losartan decreases serum uric acid in hypertensive patients via inhibiting urate transporter 1.

- Uricosuric action of losartan is different in patients and the mechanism of its individual uricosuric action is still not clear.

\section{Patients \& methods}

- A total of 101 hypertensive patients with hyperuricemia were genotyped to undergo a 2-weeks' losartan treatment. Patients were prescribed oral losartan (100 mg/day) for 2 consecutive weeks.

- Before and after treatment, serum uric acid (SUA) and other clinical data were compared between different genotypes of URAT1 patients.

Results

- The frequency of rs3825016 (C/T) CT genotype was significantly higher in the hypertensive patients with hyperuricemia than that in the healthy controls ( 32.7 vs $18.8 \% ; p=0.02$ ).

- After lorsatan treatment, the patients with the rs3825016 (C/T) or rs1529909 (T/C) mutant genotypes had lower decreased value (DV) of SUA compared with the patients who are wild-type of the variant $(p=0.001$ and $p<0.001$, respectively). Combined the two variants together, the DV of SUA in two variants both wildtype patients higher than that in the two variants mutant patients $(p<0.0001)$.

\section{Conclusion}

- The rs3825016 (C/T) and rs1529909 (T/C) variants of URAT1 influence the uricosuric action of losartan in hypertensive patients with hyperuricemia. They are potential genetic markers for the optimization of losartan's uricosuric action.

\section{References}

1 Hamada T, Hisatome I, Kinugasa Y et al. Effect of the angiotensin II receptor antagonist losartan on uric acid and oxypurine metabolism in healthy subjects. Intern. Med. 41(10), 793-797 (2002).

2 Nakashima M, Uematsu T, Kosuge K, Kanamaru M. Pilot study of the uricosuric effect of DuP-753, a new angiotensin
II receptor antagonist, in healthy subjects. Eur. J. Clin. Pharmacol. 42(3), 333-335 (1992).

3 Puig JG, Mateos F, Buno A, Ortega R, Rodriguez F, Dal-Re R. Effect of eprosartan and losartan on uric acid metabolism in patients with essential hypertension. J. Hypertension 17(7), 1033-1039 (1999).

4 Schmitt F, Natov S, Martinez F, Lacour B, Hannedouche TP. Renal effects of angiotensin I-receptor blockade and 
angiotensin convertase inhibition in man. Clin. Sci. 90(3), 205-213 (1996).

5 Rayner BL, Trinder YA, Baines D, Isaacs S, Opie LH. Effect of losartan versus candesartan on uric acid, renal function, and fibrinogen in patients with hypertension and hyperuricemia associated with diuretics. Am. J. Hypertension 19(2), 208-213 (2006).

6 Enomoto A, Endou H. Roles of organic anion transporters (OATs) and a urate transporter (URAT1) in the pathophysiology of human disease. Clin. Exp. Nephrol. 9(3), 195-205 (2005).

7 Iwanaga T, Sato M, Maeda T, Ogihara T, Tamai I. Concentration-dependent mode of interaction of angiotensin II receptor blockers with uric acid transporter. J. Pharmacol. Exp. Ther. 320(1), 211-217 (2007).

8 Burnier M, Roch-Ramel F, Brunner HR. Renal effects of angiotensin II receptor blockade in normotensive subjects. Kidney Int. 49(6), 1787-1790 (1996).

9 Enomoto A, Kimura H, Chairoungdua A et al. Molecular identification of a renal urate anion exchanger that regulates blood urate levels. Nature 417(6887), 447-452 (2002).

10 Roch-Ramel F, Guisan B, Diezi J. Effects of uricosuric and antiuricosuric agents on urate transport in human brushborder membrane vesicles. J. Pharmacol. Exp. Ther. 280(2), 839-845 (1997).

11 Alderman MH, Cohen H, Madhavan S, Kivlighn S. Serum uric acid and cardiovascular events in successfully treated hypertensive patients. Hypertension 34(1), 144-150 (1999).

12 Nindita Y, Hamada T, Bahrudin U et al. Effect of losartan and benzbromarone on the level of human urate transporter 1 mRNA. Arzneimittelforschung 60(4), 186-188 (2010).

13 Roch-Ramel F, Werner D, Guisan B. Urate transport in brush-border membrane of human kidney. Am. J. Physiol. 266(5 Pt 2), F797-F805 (1994).

14 Jutabha P, Kanai Y, Hosoyamada M et al. Identification of a novel voltage-driven organic anion transporter present at apical membrane of renal proximal tubule. J. Biol. Chem. 278(30), 27930-27938 (2003).

15 Hamada T, Ichida K, Hosoyamada M et al. Uricosuric action of losartan via the inhibition of urate transporter 1 (URAT 1) in hypertensive patients. Am. J. Hypertension 21(10), 1157-1162 (2008).

16 Fujimori S, Oka Y, Ogata N, Eto K. Effects of losartan/ hydrochlorothiazide on serum uric acid levels and blood pressure in hypertensive patients. Nucleosides Nucleotides Nucleic Acids 30 (12), 1030-1034 (2011).
17 Sui X, Church TS, Meriwether RA, Lobelo F, Blair SN. Uric acid and the development of metabolic syndrome in women and men. Metabolism 57(6), 845-852 (2008).

18 Sweet CS, Bradstreet DC, Berman RS, Jallard N, Saenz A, Weidler DJ. Pharmacodynamic activity of intravenous E-3174, an angiotensin II antagonist, in patients with essential hypertension. Am. J. Hypertension 7(12), 1035-1040 (1994).

19 Dang A, Zhang Y, Liu G, Chen G, Song W, Wang B. Effects of losartan and irbesartan on serum uric acid in hypertensive patients with hyperuricaemia in Chinese population. J. Hum. Hypertension 20 (1), 45-50 (2006).

20 Hosoya T, Kuriyama S, Yoshizawa T, Kobayashi A, Otsuka Y, Ohno I. Effects of combined antihypertensive therapy with losartan/hydrochlorothiazide on uric acid metabolism. Intern. Med. 51(18), 2509-2514 (2012).

21 Graessler J, Graessler A, Unger S et al. Association of the human urate transporter 1 with reduced renal uric acid excretion and hyperuricemia in a German Caucasian population. Arthritis Rheum. 54(1), 292-300 (2006).

22 Iwai N, Mino Y, Hosoyamada M, Tago N, Kokubo Y, Endou H. A high prevalence of renal hypouricemia caused by inactive SLC22A12 in Japanese. Kidney Int. 66(3), 935-944 (2004).

23 Jang WC, Nam YH, Park SM et al. T6092C polymorphism of $S L C 22 A 12$ gene is associated with serum uric acid concentrations in Korean male subjects. Clin. Chim. Acta 398(1-2), 140-144 (2008).

24 Wurzner G, Gerster JC, Chiolero A et al. Comparative effects of losartan and irbesartan on serum uric acid in hypertensive patients with hyperuricaemia and gout. J. Hypertension 19(10), 1855-1860 (2001).

25 Ohshiro K, Sakima A, Nakada S et al. Beneficial effect of switching from a combination of angiotensin II receptor blockers other than losartan and thiazides to a fixed dose of losartan/hydrochlorothiazide on uric acid metabolism in hypertensive patients. Clin. Exp. Hypertension 33(8), 565-570 (2011).

26 Hamada T, Mizuta E, Kondo T et al. Effects of a low-dose antihypertensive diuretic in combination with losartan, telmisartan, or candesartan on serum urate levels in hypertensive patients. Arzneimittelforschung 60 (2), 71-75 (2010).

27 Shima Y TK, Ohta H. Association between intronic SNP in urate-anion exchanger gene, $S L C 22 A 12$, and serum uric acid levels in Japanese. Life Sci. 79(23), 2234-2237 (2006). 